\title{
Supporting Map-Based Wayfinding with Tactile Cues
}

\author{
Martin Pielot \\ OFFIS Institute for Information \\ Technology \\ Oldenburg, Germany \\ pielot@offis.de
}

\author{
Niels Henze \\ OFFIS Institute for Information \\ Technology \\ Oldenburg, Germany \\ henze@offis.de
}

\author{
Susanne Boll \\ University of Oldenburg \\ Oldenburg, Germany \\ boll@informatik.uni- \\ oldenburg.de
}

\begin{abstract}
Paper maps are a proven means for navigating in unfamiliar environments, however, they do not prevent people from getting lost or taking unwanted detours. A well-known issue is interpreting the map's geocentric content, which is known to become prone to errors when the map is not aligned to the environment. In this paper we report our investigation of providing a cue about the destination's location from an egocentric perspective in order to improve the interpretation of the map. We used a vibrotactile belt to continuously indicate a destination's direction relative to the user's orientation. In an outdoor field study we compared the performance of map-based navigation with and without the added tactile cue. We found evidence that people take shorter routes, consult the map less often, and were less often disoriented with the tactile cue. Furthermore, females found the tactile cue more useful and used it more often.
\end{abstract}

\section{Categories and Subject Descriptors}

H.5.2 [User Interfaces]: Haptic I/O; I.3.6 [Methodology and Techniques]: Interaction techniques

\section{General Terms}

Human Factors, Experimentation

\section{Keywords}

tactile display, pedestrian navigation, wayfinding

\section{INTRODUCTION}

Maps as one of the oldest known information artefacts are used for over 5000 years [4]. They are used to acquire spatial knowledge, orient oneself in the environment, and navigate between places. At the end of the last century the development of geographic information systems, global navigation satellite system, and powerful mobile devices allowed the development of personal navigation devices (PNDs) providing us with map data at our fingertips.

However, paper maps still have many advantages over PNDs with respect to pedestrian navigation. They allow pedestrians to define their own routes, find shortcuts and alternative routes, and while strolling around without a specific destination they help remaining oriented. They provide

Copyright is held by the author/owner(s).

MobileHCI'09 September 15-18, 2009, Bonn, Germany ACM 978-1-60558-281-8.

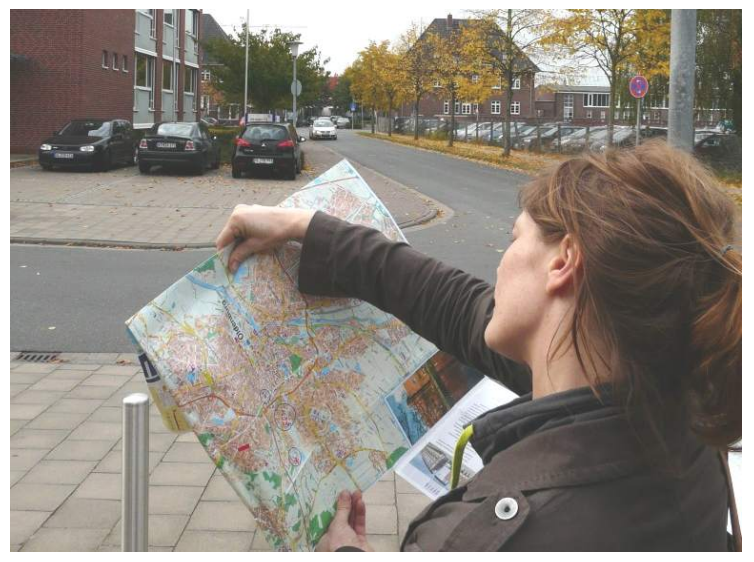

Figure 1: A pedestrian who is physically aligning the map to the environment.

survey knowledge which in contrast to route-instructions as conveyed by PNDs results into a better knowledge about a geographic environment [2]. Survey knowledge is also more robust, since it allows recovering from navigation errors and finding alternative routes [6]. Nevertheless, interpreting paper maps and applying the survey knowledge to the surrounding environment is not a trivial task.

PNDs take away the burden of interpreting the geocentric spatial information. They show the user's location and the planned route on an interactive map. Additionally, turn-byturn route instructions are conveyed visually and via speech output, such as 'in fifty meters turn left'. However, paper maps still outperform commercial PNDs in many aspects. Providing route-instructions has shown to disengage users from the environment [7] and impede understanding the spatial layout of the environments [2]. Using distances to located turning points also contradicts the way people naturally describe routes, as their descriptions mainly comprise landmarks $[9,13,3]$. Consequently, field studies indicate that paper maps are favoured over PNDs and allow a more efficient navigation $[5,14]$.

A known issue related to paper maps is that the map's geocentric spatial information has to be interpreted and aligned to the environment [1]. This is called the alignment effect. It can be countered by physically aligning the map with the environment, as shown in Figure 1. Doing so eases their interpretation [15]. In this paper we investigate a different approach to simplify the interpretation of paper maps. Instead of rotating the map physically we support its mental 
rotation by continuously conveying the direction of a trip's destination. This is done by a tactile display consisting of several tactile actuators (tactors). It is worn around the user's waist similar to a belt. Regardless of the user's location and orientation it always indicates the destination's location, by activating the tactor that points most accurately into its direction. Previous research shows that people easily can interpret the directions provided by such tactile displays $[18,23,20]$.

In an outdoor field study we evaluated how using the tactile display affects navigation with a paper map. We present evidence that the tactile display causes people to take shorter routes, be less distracted by the map, and loose their orientation less often.

\section{RELATED WORK}

Knowledge about space can be preserved in an egocentric (the observer's perspective) form, or a geocentric form (such as found in paper maps). If geocentric spatial knowledge, e.g. a paper map, shall be applied to a real-world task, such as navigation, it has to be interpreted. When interpreting paper maps, people tend to make fewer mistakes when the map rotation corresponds to the user's forward direction. This effect is called the "Alignment effect" and is attributed to the mental rotation, that has to be done by the map reader [1]. Rotating the map automatically according to the movement direction has been shown to improve the navigation efficiency [15].

Tactile displays that convey spatial information for improving the sense of orientation $[21,10,23]$ or the situation awareness $[8,16]$ have been investigated for quite a while. Tactile belts, such as the ActiveBelt by Tsukada and Yasumura [18], consist of a number of tactors (e.g. eight) that are attached to a belt or a vest. If the belt is worn the tactors are equally distributed around the user's body. Tactors can then be activated to point into directions. Van Erp et al. [21] showed that providing egocentric information about the current orientation through such a tactile display can help people countering a spatial disorientation event. Participants were rotated on a chair and had to control the rotation until they stood still. The tactile cue significantly reduced the time the participants needed to stop the rotation. Nagel et al. [10] investigated if such tactile feedback can become an additional sense. After six weeks of wearing a tactile belt displaying north, participants from the experimental group showed signs of having the feedback integrated into their normal senses. Participants who had embraced the new technology reported a substantial change of their perception of the environment.

Displaying the directions of locations, e.g. waypoints, has been investigated as well. Van Veen et al. [23] has shown that people can easily follow routes if the direction of the current waypoint is being displayed by a tactile belt. Lindeman et al [8] could show that displaying the presence of hazardous spots in around the user improves the situation awareness of soldiers in a simulated building-clearing task. Participants were more effective in avoiding exposure to uncleared places. However, the exposure only reduced in relation to the completion time when using the tactile belt. While the total completion time increased significantly, the absolute time (in seconds) being exposed was roughly the same. Smets et al. [16] combined the use of a tactile vest for displaying the destination's direction with head-up and north-up maps. They conducted a study in a virtual environment, where the participants had to reach given places as fast as possible. The results showed that the egocentric tactile cue could reduce the mental effort required for interpreting a north-up map so the completion times were at a par with those where a forward-up map was provided.

Those results indicate that providing a tactile belt in addition to a paper map could counter the alignment effect and improve the general navigation performance. However, no study has yet investigated providing a directional tactile cue in a real-world navigation scenario. The results by Smets et al. will not automatically apply to real-world navigation, since real world studies with navigation aids showed an unexpectedly bad navigation performance with paper maps and PNDs [5]. Additionally it has not yet been shown that the understanding of the environment is improved by the added tactile cue.

\section{TACTILE PROTOTYPE DESIGN}

In order to reach a destination with a paper map the user has to stay orientated. A paper map provides information about the environment, the location of the destination, and possible paths. But it offers little help in determining one's position and orientation in relation to these spatial entities. Providing route instruction, such as navigation systems do, would solve that situation, however, it would prevent people from strolling around freely in the environment.

We therefore propose to convey the real world location of a spatial entity that is also shown in the map. We suppose that cueing a direction from the egocentric point of view helps to mentally rotate the map's geocentric content accordingly. In this work we presented the direction of the destination as orientation aid, as it would be most helpful. This concept is visualised in Figure 2.

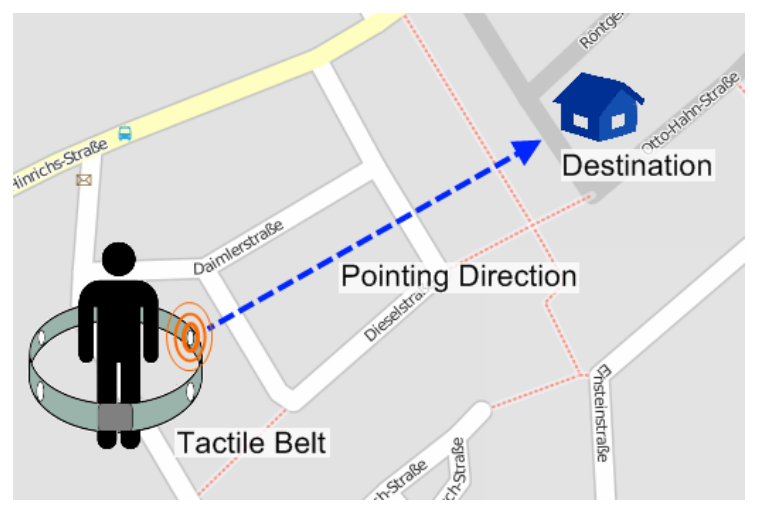

Figure 2: Pointing at the trip's destination with a tactile belt for assisting navigation with a paper map.

Previous work indicates that conveying directions of waypoints with tactile displays allows people to reach them easily. Thus, it can be assumed that people are able to interpret the direction of a reference spatial entity as well. Conveying distances of waypoints has also been investigated but not shown any significant effect on the effective walking speed $[22,23]$. Thus, we focus on presenting the direction of a spatial entity. This section describes information presentation design, the tactile display design, and the application 
we used to cue the direction of a trips destination to the user.

\subsection{Tactile Direction Cueing}

To provide directions to the user a display is needed that is usable in an outdoor environment while being on the move. As described in the previous section visual displays and auditory displays have limitations if used outdoors. Especially in our case, where people are expected to navigate in an unfamiliar environment and handle a paper map at the same time, the information presentation needs to occur eyes-free and hands-free. Impairing the auditory sense would limit the experience of the environment, which can even be dangerous in traffic-rich streets. In addition, during a field study it impedes the communication between the experimenter and the participants, so less verbal comments can be expected. Using the sense of touch for communication does not overlay the user's visual or audio senses. We therefore used a tactile display for conveying the destinations direction. In addition, tactile displays can easily be mounted so that the hands remain free.

The most frequent used tactile displays for conveying directions are tactile belts or vests where the actuators are distributed around the torso. Many studies have shown that people are able to intuitively externalise directions from stimulation around the body $[20,18,8]$. In those prototypes, the tactor that points closest into the direction to be displays is activated displayed (see Figure 3). Depending on the location of the tactors, stimuli can be produced all around the user. For example, a stimulus on the back near the spine is perceived as "behind" or 6 o'clock. A stimulus on the abdomen right under the navel is intuitively mapped to "front" or 12 o'clock.
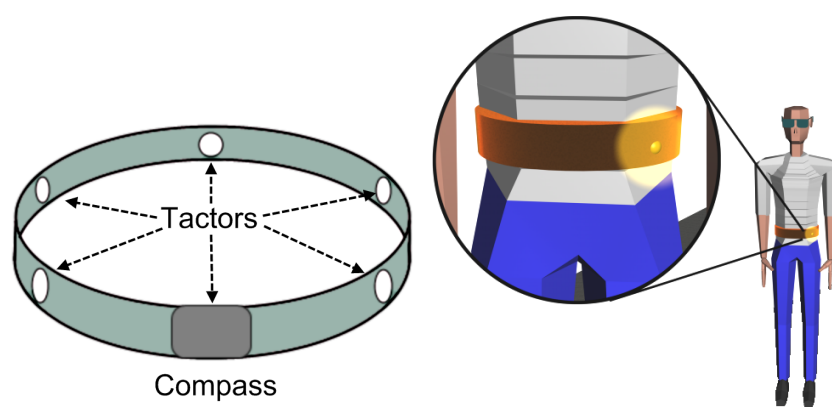

Figure 3: Sketch of the tactile belt (left), egocentric direction cueing with the tactile belt (right)

Common approaches $[18,23,22,8]$ map a range of directions to single tactors by activating the tactor that is closest to the direction to be displayed. This limits the resolution of the devices to the number of actuators. However, as the human perception is not perfect, spatial effects of tactile perception can be exploited to generate the impression of stimuli at locations where not actuators are. Tan and Pentland [17], for example, utilized the sensory saltation effect. Three actuators attach along the arm in a row are activated in succession where each actuator produced three short stimuli. The user perceives each pulse in a different location, continuously wandering along the arm. However, due to the moving nature of the perceived signal this approach can not be combined with the mapping of a tactile stimulus at the waist to a horizontal direction described above. In our previous work [11] we investigated the exploitation of the apparent location effect [19]. This effect describes the phenomenon that two tactile stimuli are perceived as one when they occur locally close together. The perceived location of the stimulus depends on the relative magnitude of both actual stimuli. As shown in Figure 4, we used two adjacent tactors to produce an apparent stimulus in between them. Thus, it theoretically would be possible to produce a continuous sensation around the body.

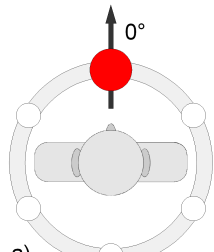

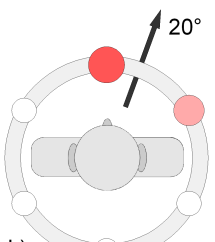

b)

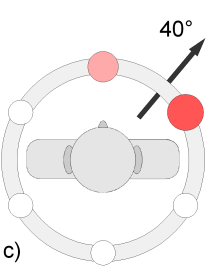

c)

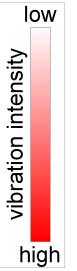

Figure 4: Visualisation of the interpolating presentation technique [11]: two actuators at $0^{\circ}$ and $60^{\circ}$ are used to exemplarily display three directions $\left(0^{\circ}\right.$, $20^{\circ}$, and $40^{\circ}$ ).

If the skin is exposed to tactile stimulation it will adapt to it, making the tactile stimulus harder to perceive [19]. Other researchers (e.g. [16]) therefore choose a pulsing display where the pauses between stimulations are sufficient for the skin to regenerate to full sensitiveness. However, stimulating the skin with pulses means that the stimulation is more obtrusive, as the perception constantly changes. The feelspace project [10] showed that a gentle, continuous stimulation with a tactile belt can be perceived over weeks without problems. They even showed that people started to process the directional hints subconsciously, giving people a much better awareness of their orientation and thus a better understanding of their surrounding. To mediate directions consciously we therefore decided to keep our stimulation continuous while reducing the overall intensity.

The immanent inaccuracy of today's outdoor localisation techniques, in particular the GPS system, can result in a significant deviation between the user's real position and the measured position. The influence of this inaccuracy on the tactile output increases as the user approaches the destination. If being too close to the destination, the inconsistent position estimation could result in a confusing output. We therefore alter the display method if the user is near the destination. In order to get the user's attention we turn the display from a continuous mode into a pulsing mode. The pulses were $500 \mathrm{~ms}$ of stimulus followed by a $500 \mathrm{~ms}$ pause.

\subsection{Hardware}

The hardware used comprises a custom built belt-like tactile display (see Fig.5). It consists of flexible fabric with six evenly distributed tactors. The used tactors have a diameter of about $11 \mathrm{~mm}$ and can also be found in Samsung SGH A400 mobile phones. With $50 \%$ of the maximal input voltage the tactors vibrate around $100 \mathrm{hz}$. They are mounted on small drilled boards as shown in Figure 6. The belt's control logic is integrated in a small box attached to the fabric. The box also contains an electronic compass and can be controlled by a integrated Bluetooth interface. To display the destination's direction relative to the user's orientation 




Figure 5: The tactile belt used for the study. The flexible fabrics houses six vibrotactile actuators which equally distribute around the torso if the belt is worn.

the system must determine the user's heading and position. The integrated electronic compass provides its absolute direction in the horizontal plane. The compass has an update rate of $100 \mathrm{~Hz}$ and an accuracy of about $2^{\circ}$. Thus, direct tactile feedback is provided if the user turns his or her body. To determine the user's position an external GPS receiver is connected to the belt. Using position and heading the direction presentation can be adjusted to the user's orientation, which enables to display absolute directions. The tactile belt

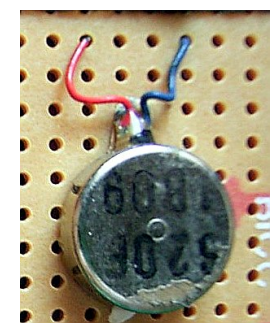

Figure 6: One of the vibrotactile actuators used in the tactile belt.

is controlled by an application running on a mobile phone. This application allows specifying a geo location as destination. The belt's direction sensor and GPS receiver are used to determine the wearer's location and orientation. By comparing the user's position with the destination's position an according vector is computed. Subtracting the direction of this vector from the user's absolute orientation leads to the direction that is displayed to the user. The distance between those two geo locations is used to determine, if the tactile stimulus is continuous (more than 50 meters) or pulsing (50 meters and less).

\section{EVALUATION}

The goal of the study was to investigate how the above described navigation aid based on the tactile belt would affect the navigation with a paper map. The study took place in a village where study's participants had to reach two given destinations. The actual route to these destinations had to be chosen by the participants themselves. Therefore, they were either equipped with the paper map and the tactile belt pointing at the respective destination or with the paper map only. We assumed that the tactile directional cue would give people a better sense of orientation, which would improve the navigation with the paper map in three ways: (H1) The participants will take more efficient routes, even if they are more complicated. (H2) The participants will be less distracted by the paper map. (H3) The participants will experience less events of disorientation.

\subsection{Environment}

The evaluation took place in a typical German village with an organically grown, unsystematic street network (see Figure 7 ). Besides the main roads it comprised many trafficcalmed streets and small pedestrian paths. We assigned a starting point and two destinations, so there were two different sessions planned. The first destination also served as starting point for the second session. The air-line distance between each starting point and the corresponding destination was about 500 meters. In both cases there was a long and easy route along the main roads which lead to the destinations. Additionally, there were plenty of potential shortcuts, mostly by taking smaller roads, pedestrian paths, or even open fields, such as playgrounds. Figure 8 shows the three places forming two sessions (get from A to B, and get from $\mathrm{B}$ to $\mathrm{C}$ ). We did not provide the participants with any suggestions about what route to take. Thus, each participant had to find her or his own path to the destinations.

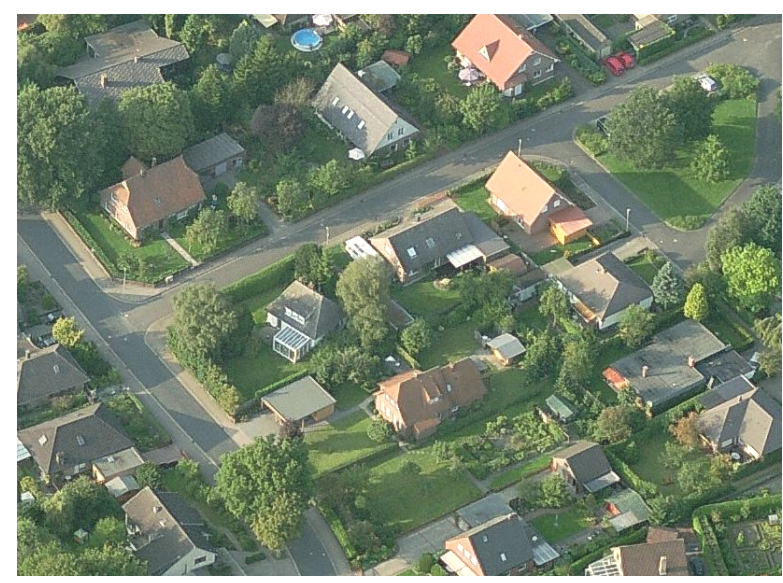

Figure 7: The typical German village with single family houses and an organically grown street network where the evaluation took place (from http://maps.live.com).

\subsection{Paper Map}

As the primary navigation aid we provided the participants with paper maps that fully covered the evaluation environment. The map's size is about one square meter unfolded and the scale is 1:22500. It shows all roads and pedestrian paths in the environment. Except for the pedestrian paths all streets were labelled with the respective street name. The evaluation environment was printed directly onto a fold, so the map had to be at least partially unfolded to look at the whole village at once. The three places A,B, and $\mathrm{C}$ were marked with small dots. Figure 9 a participants studying the provided paper map.

During pilot studies we discovered that the maps were slightly inaccurate in some places. For example, Figure 10 shows a pedestrian path which is denoted as a road on the 


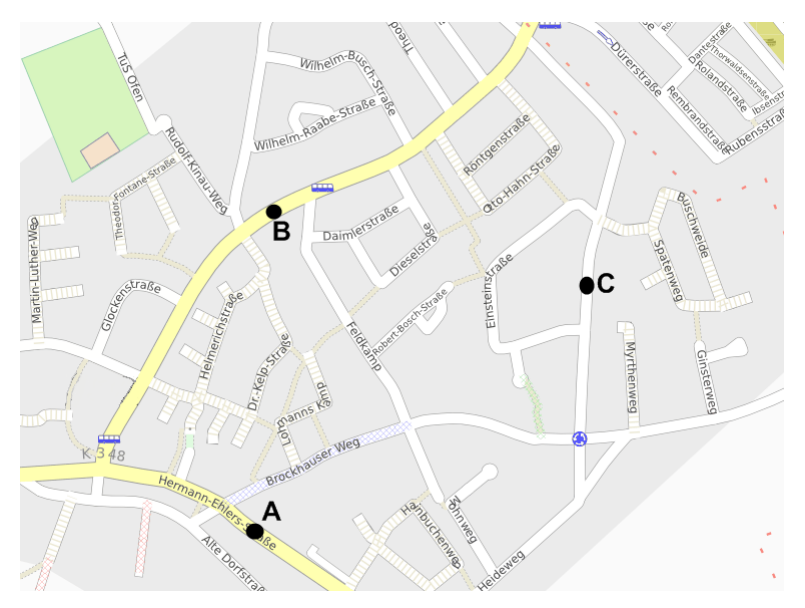

Figure 8: Overview of the evaluation environment: Participants started at A. Then had to reach $B$ and from there go to $C$ (from http://www.openstreetmap.org/).

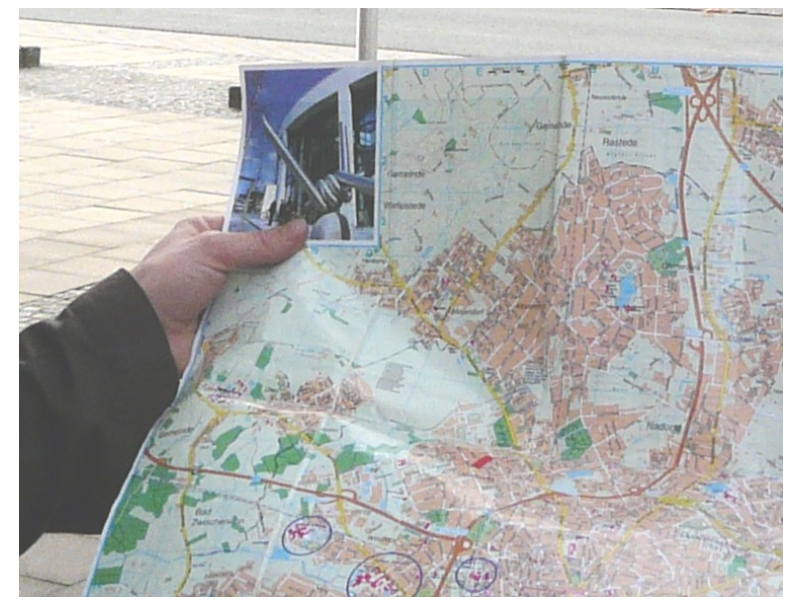

Figure 9: The paper map that was used in the study. The evaluation environment is located near the upper-left corner of the map.

paper map. Several of these inaccuracies existed along potential routes the participants could take, so they could possibly cause the participants to loose confidence about the chosen path.

\subsection{Design}

In the control condition, participants received the paper map only as navigation aid. In the experimental condition we additionally provided them with the tactile belt that was configured to point towards the session's destination. The study used a repeated-measures design. The order of conditions was counterbalanced amongst the participants. Eight participants started in the experimental condition and the other eight in the control condition. In each of these groups of eight participants the gender was equally split. In order to recognise any possible sequence effects, the scores of the group that started in the control condition and the group that started in the experimental condition were checked for significant differences.

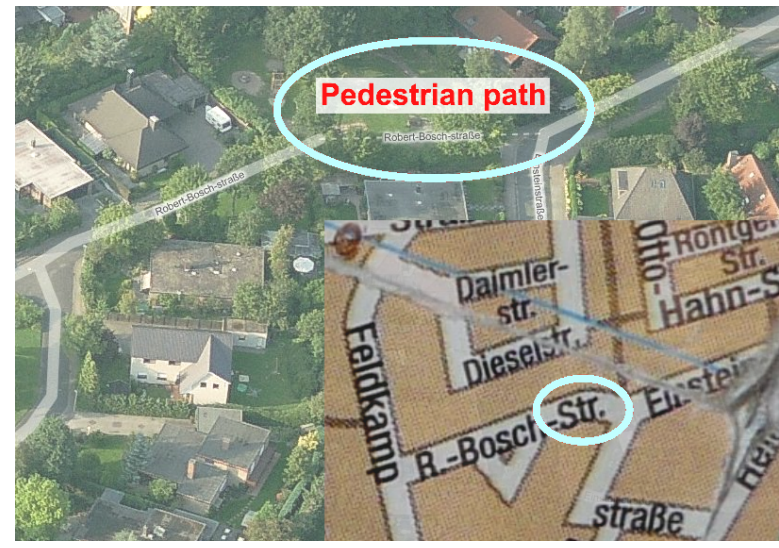

Figure 10: Example of the paper map's inaccuracy: a pedestrian path is shown as a street on the map (air photo from http://maps.live.com).

\subsection{Dependent Measures}

We used GPS trackers and video cameras to record the participants' movement, comments, and actions during the evaluation. The GPS trackers allowed us to reconstruct the route each participant had walked. The video recordings allowed us to investigate the behaviour of the participants. The participants were encouraged to think aloud so their thoughts and comments were recorded on the video as well. The following dependent measures were taken:

\section{Efficiency.}

The completion time, route length, and effective walking speed were measured as an indicator for the effectiveness of the provided navigation aids. Due to the layout of the evaluation environment, a shorter route meant that a participant had to deviate from the main roads and take side roads and pedestrian paths which were more complicated and unclear.

\section{Distraction.}

For quantifying how much the participants were distracted by the paper map and the tactile belt we measured how often and how long they interacted with them. Interacting with the map was defined as people were holding the map in their field of vision. Interacting with the tactile belt was defined as conscious, visible interaction with the device, e.g. by turning the hip slightly in order to alter the tactile signal, or expressing the tactile perception to the experimenter.

\section{Disorientation.}

Disorientation events were defined as situations where participants stopped and either stated that they felt disoriented, or when they began to study the map and the environment for more than ten seconds while not moving into any specific direction. When participants were wearing the tactile belt and experienced a disorientation event, we also noted if the tactile belt was used to resolve the loss of orientation. We counted only those occurrences where participants explicitly stated that they based their decision about how to proceed based on the tactile cue.

\section{Perceived Helpfulness.}

After the participants had reached both destinations they 
were asked to rate the paper map and the tactile belt about how helpful they found each navigation aid for orienting themselves and how much they relied on it for choosing a route. Each of the four aspects were rated on a five-point Likert scale, where 5 corresponded to not helpful and 1 corresponded to very helpful.

\subsection{Participants}

Sixteen participants, 8 female and 8 male, took part in the user study. They were aged between 20 and 33 (Mean $=26.88)$. None of them was familiar with the environment where the evaluation took place.

\subsection{Procedure}

The evaluation was carried out individually. Each participants had to get from place A to place B and then from place B to place C (see Figure 8. Initially, the experimenter explained the relevant features of the paper map and highlighted the locations of the starting point and the two destinations to the participants. The participants also learned how different types of roads were presented on the map. Next, the participants were familiarized with the tactile belt and how directions were presented. Then, as training session each participant had to use a paper map and the tactile belt to navigate from the parking site to the first starting point (A). This ensured, that the participants where already oriented at the beginning of both trips. Otherwise, the initial lack of orientation would have affected the results from the first route negatively. If the participants started in the control condition the belt was taken off at this point. Otherwise it was configured to display the direction of the first destination (B). The participants were then asked to find the first destination with the help of the given navigation aids. No hints about the route or the environment were given. The experimenter followed the participants with the video camera in a short distance, focussing on their interaction with the navigation aids and the environment. Once the participant reached the first destination, the conditions were switched, and the participant was asked to reach the second destination (C). Having arrived, the participants were asked to rate the map and the tactile belt regarding their helpfulness for orientation and navigation.

\section{RESULTS}

The video recordings and the recorded GPS tracks were used to retrieve the qualitative and quantitative results. The videos were analysed with the help of a custom built application. To accurately log the occurrences of predefined events and their duration, the experimenters had to press keys as long as these events occured (see Dependent Measures 4.4 for definitions). One key had to be pressed as long as the participant observably looked at the map and another key as long as the participant observably interacted with the belt. A third key was pressed each time the participant was disoriented. In addition, the videos were used to determine each participant's travel time.

\subsection{Quantitative Results}

In the following the results regarding the dependent measures are given. In addition, we report unexpected significant findings related to the participants' gender. The results are visualized in diagrams showing the mean in each condition over all participants and the $95 \%$ confidence intervals.
In the legends of the diagrams map denotes the results from the control condition (paper map only) and mapÉbelt denotes the results from experimental condition (paper map and tactile belt). The differences are significant at a level of at least $\mathrm{p}<.05$ unless stated otherwise. In addition to the $\mathrm{t}-$ test results, the $r$-value are provided denoting the Pearson's correlation and thus indicating the effect size.

The repeated-measures design we used in our study is known to be vulnerable to sequence effects, such as increasing practice or fatigue, since participants repeat tasks. In order to ensure that our findings were not biased by sequence effects we checked the results from all participants first trips to all participants second trips for significant differences. Additionally, we compared the results from participants who started in the control condition with those how started in the experimental condition. No sequence effects were found.

\subsubsection{Efficiency}

The efficiency of the navigation aids was measured in terms of route length, walking speed, and completion time. The recorded GPS tracks were used to reconstruct the route that each participant had taken within Google Maps. These reconstructed routes were used to obtain the length of the travelled route without adding the variance of the GPS signal. The time needed to complete each route was taken from the videos. Route length and completion time were used to calculate the effective average walking speed for each trip. The results are given in the following paragraphs:

The travelled distance decreased significantly with the tactile belt $(\mathrm{t}(15)=1.93, \mathrm{p}<.05, \mathrm{r}=-.009)$.

\section{Average route length of each condition}

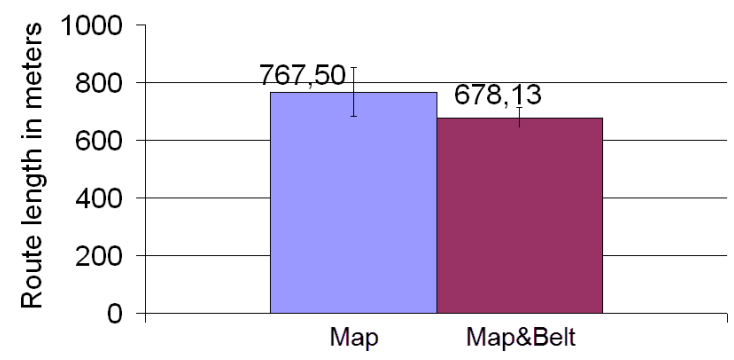

Figure 11: The participants took shorter routes when using the tactile belt in addition to the paper map.

Figure 12 depicts the average walking speed for each condition. The walking speed decreased significantly with the tactile belt $(\mathrm{t}(15)=3.39, \mathrm{p}<.01, \mathrm{r}=.342)$.

There was no significant effect on the completion time. The average travel time for each condition is shown in Figure 13.

In summary, the participants walked slower with the tactile belt but took shorter, more efficient routes, which in the end did not significantly affect the travel time.

\subsubsection{Distraction}

The distraction was measured in terms of how often and how long the participants looked at the paper map. As illustrated above, these measures were taken from the video recordings. We only report the average time the participants 
Walking Speed

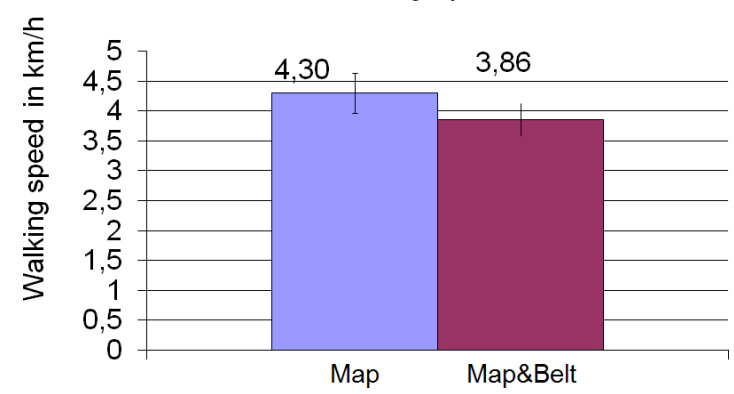

Figure 12: The participants walked faster when only using the paper map.

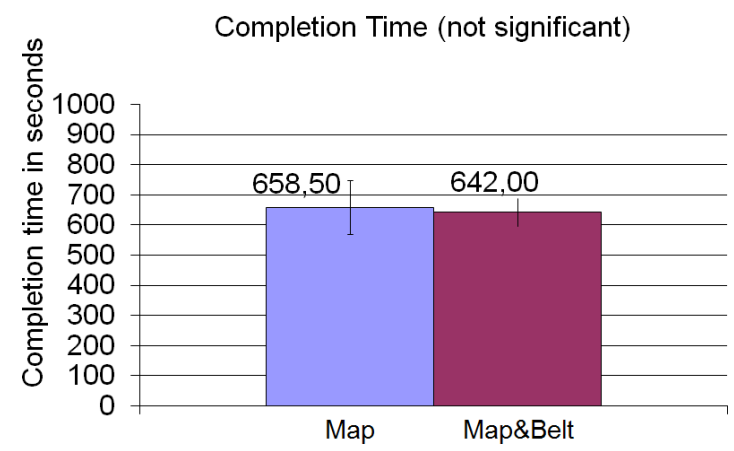

Figure 13: The average travel time did not significantly differ between the conditions.

spent looking at the map, since both scores were highly correlated. The time spend looking at the map was normalized by dividing it by the completion time. The resulting values are the fractions of the travel time each participant spent looking at the map.

The average time for each condition is shown in Figure 14. The average time spend looking at the map was significantly reduced with the tactile belt $(\mathrm{t}(15)=5,53, \mathrm{p}<.001, \mathrm{r}=$ $.743)$.

Time spent looking at the map

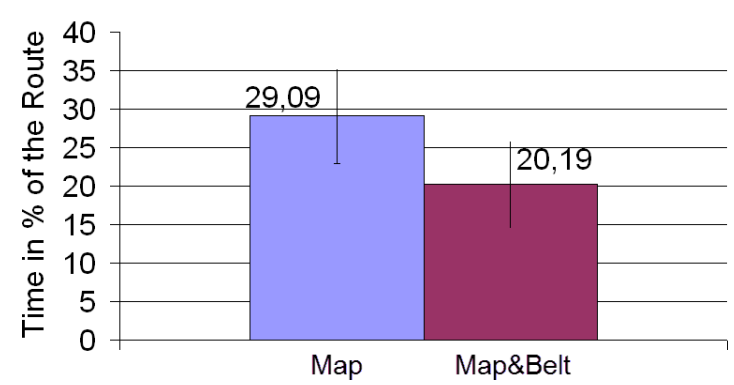

Figure 14: The participants consulted the paper map less often when equipped with the tacile belt.

The average time people spend interacting with the tactile belt was $10.29 \%$. If this number is added to the time spend looking at the map in the experimental condition, there was no significant difference between the conditions. This means that the participants interacted about the same time with one of the available navigation aids.

\subsubsection{Disorientation}

The disorientation was measured in terms of how often a participants lost her or his orientation according to the criteria outlined in Section 4.4. As the number of occurrences and the time were highly correlated we report the number of disorientation events only. The average number of disorientation events for calculated by summarizing all occurrences for each condition and divide the result by the number of participants.

Occurences of disorientation events

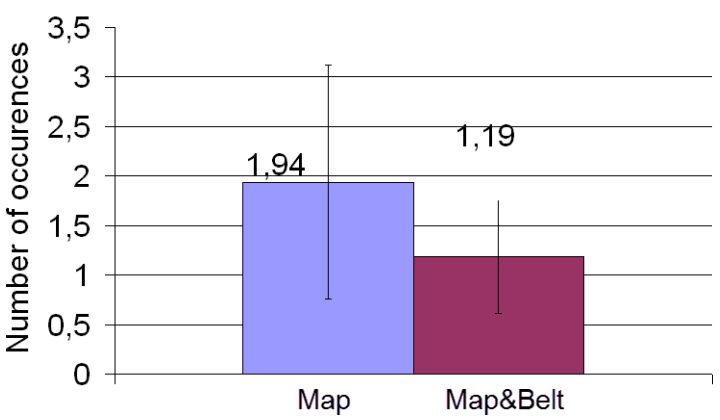

Figure 15: The tactile belt reduced the number of disorientation events.

The average number of disorientation events in each condition is shown in Figure 15. The number of disorientation events significantly decreased with the tactile belt $(\mathrm{t}(15)=$ $1.78, \mathrm{p}<.05, \mathrm{r}=.787)$.

In the experimental condition, in twelve out of nineteen cases of disorientation, the participant spontaneously reported that she or he was using the tactile belt to resolve their disorientation.

\subsubsection{Subjective helpfulness}

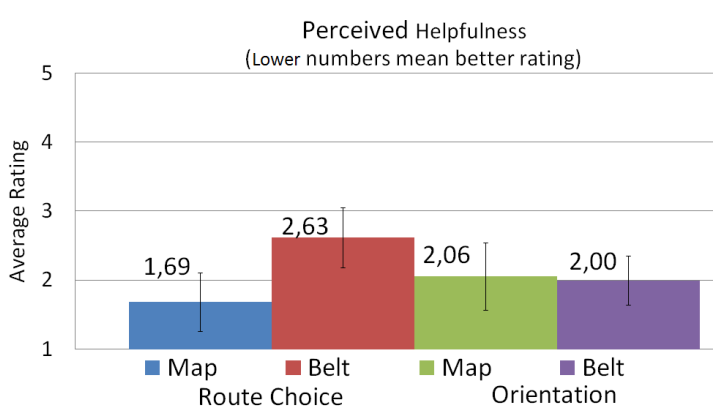

Figure 16: The map was rated significantly more helpful than the tactile belt for planning the route. Both navigation aids were rated equally regarding the helpfulness for orienting oneself in the environment.

Participants found the paper map significantly more helpful for planning and choosing their route. The paper map and the tactile belt were both rated to be helpful for staying oriented. No significant difference could be found. The average ratings for both aids in both aspects, resulting into four ratings, are shown in Figure 16. 


\subsubsection{Gender aspects}

Besides the effects of adding the tactile belt to the paper map we also observed differences between the genders. Since both genders were equally represented and equally split amongst the order of conditions, we were able to analyze the data in a meaningful way.

Figure 17 shows how helpful each gender rated the tactile belt for staying oriented. Female participants rated the belt significantly more useful for orienting themselves in the environments than male participants $(\mathrm{t}(15)=2.15, \mathrm{p}<.05$, two-sided, $\mathrm{r}=-0.33$ ).

Perceived usefulness of tactile belt for orientation (smaller number is better rating)

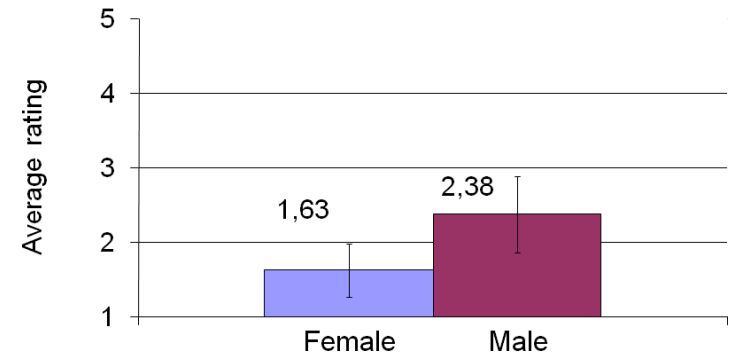

Figure 17: Females found the belt more useful for orientation than males.

Figure 18 shows the average normalized time each gender spend interacting with the tactile belt. Female participants interacted significantly more often with the tactile belt than males $(\mathrm{t}(15)=4.23, \mathrm{p}<.001$, two-sided, $\mathrm{r}=0.35)$.

\section{Time spend interacting with the tactile belt}

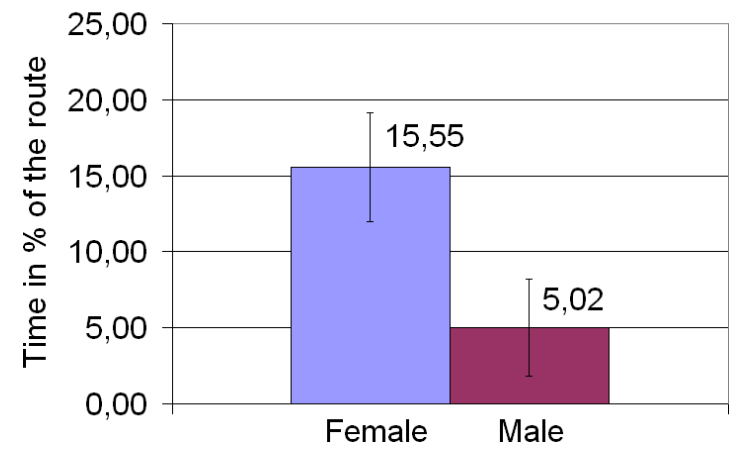

Figure 18: Females interacted more often with the tactile belt than males.

In summary, female rated the belt to be more helpful and made more use of it.

\subsection{Observations and Comments}

In this section we describe qualitative findings that were mainly taking from observations and comments recorded in the videos. We structured the findings by the aspects of map usage, tactile belt usage, and integration of both navigation aids.

\section{Map usage.}

As the quantitative results show, the participants spend roughly $20 \%$ to $30 \%$ of the whole trip looking at the map.
The participants not only consulted the map at decision points but looked at it very frequently. Some participants even spent about half of their trip's time looking at the map. While it is known that people rotate maps to align it with the walking direction, our participants rarely rotated the map. Those rotations were mostly limited to angles below 90 degrees, so the participants did not have to change the position of their hands. Street signs turned out to be very important for using the map with confidence. The participants used them to reassure themselves that they were still on the planned route. When there were no street signs, e.g. when a pedestrian path ended in the middle of a road, the participants quickly lost their confidence. The participants then often complained about the absence of street signs. One participant even took a detour to find a street sign, although he was relatively sure that he was still oriented. The most common reason for disorientation was the map being not congruent with the environment. Since the participants mainly relied on the map for route choice, they often neglected those pedestrian paths that were not correctly printed on the map. Therefore, some shortcuts were missed. At one place, a road turned into a pedestrian path but was still shown as a road on the map. At this place, many participants got confused. This was the situation where most people stated that they would just follow the pedestrian path, since the tactile belt was pointing into its direction.

\section{Tactile belt usage.}

The belt was rarely used at decision points for deciding how to proceed. Instead, participants used the belt to ensure that they were still on the right way. This also included resolving disorientation events. The belt helped people to regain confidence that choosing a certain route will bring them closer to the destination regardless of being disoriented. While many related tactile systems in order to avoid overstimulation employ short pulses for displaying directions only, we could not observe any negative effect of our continuous tactile stimulus. Participants were rather complaining that the tactile stimulus was too weak in general.

\section{Integration of tactile belt and paper map.}

There was a gap between how easy participants found it to integrate the information provided by the tactile belt and by the paper map. Some participants found the belt to be a good complement to the map, mentioning that the tactile cue helped them to mentally rotate the map according to the environment. In other cases, participants were confused by using map and belt in combination, stating that they could not match the different kind of information provided by both aids. Many of these participants then started to rely on one of the navigation aids only, mostly the belt. One participant did not use the map at all right from the beginning. Instead she totally relied on the directions given by the belt. Her strategy was to follow the direction cue as accurate as possible causing her in one case to climb over a fence to cross a children's playground.

\section{DISCUSSION}

In summary the directional tactile cue affected the participants' navigation behaviour: they took shorter routes, spent less time studying the map, and were less often disoriented. The paper map was perceived significantly for helpful for the task of choosing the route while for maintaining a sense of orientation both navigation aids were at a par. In addition, 
females valued the tactile belt more helpful and used it more often than males.

Hypothesis 1 (the directional cue will improve the efficiency of the navigation) is supported by the finding that the tactile belt significantly reduced the average route length. Reducing the overall route length required the participants to move closer to the airline. Taking the main roads, however, meant accepting detours from the airline. The participants had to take smaller routes or pedestrian paths instead. Since way finding based on survey knowledge (e.g., the paper map) relies on being spatially oriented [12] the in average shorter routes indicate that the participants had a better spatial orientation when provided with the tactile cue. This also supports the findings by Smets et al [16] where participants in a virtual world navigated more effectively with a vibrotactile directional cue of the destination. Nevertheless, the walking speed decreased significantly while the completion time was not significantly different with the tactile belt. We believe that people walked slower for two reasons: First, the tactile belt did not work perfectly well, so we had to stop in a few cases to check for potential malfunctions. Second, the participants took their time to study the tactile perception. As we did not ask them to complete the route as fast as possible, they did not feel in a hurry. Thus, we believe that a more robust device and some more familiarisation would lead to better completion times. However, we are not sure, if additional training and exercise with a tactile belt will reduce these kind of stops ultimately.

Hypothesis 2 (participants will be less dependent on the map) is supported by the finding that the participants looked significantly less often at the map when being equipped with the tactile belt. In fact, the tactile belt seemed to replace parts of the interaction with the paper map. There was no significant difference between the time spend looking at the map in the control condition and the time spend interaction with either - the map or the tactile belt - in the experimental condition. In average, participants interacted roughly a quarter of the trip's time with one of the navigation aids, regardless of being at decision points or not. This supports the findings of May and Ross [9, 13] who analyzed route descriptions created by humans. In their studies the participants did not only describe how to proceed at decision points but also denoted landmarks between those decision points. May and Ross assume that people desire constant confirmation that their route choice was correct. The constant use of navigation aids that we observed in our study supports this assumption. While the tactile belt did not significantly affect the overall time that participants spend interacting with either navigation aid, replacing the use of the paper map by using the tactile belt still holds an advantage. According to Wicken's Multiple Resource Theory [24] using the rarely used tactile sense for conveying information can reduce the overall cognitive load in situations with lots of visual and auditory sensations.

Hypothesis 3 (participants will have a better sense of orientation) is supported by the finding that the tactile belt significantly reduced the number of disorientation events. Although participants mainly relied on the map for way finding, the belt could decrease the number of disorientation events significantly. About half of the disorientation events were caused by situations were the road network on the paper map did not correspond with the real world. This type of disorientation event was reduced stronger by the tac- tile belt than the other types. We therefore assume that in cases of disorientation events, where the map was not conform to the participants' expectations the tactile belt reinsured them that their mental model of the environment was correct rather then the paper map. We could confirm the results from older studies about wayfinding (e.g., Ishikawa et al. [5]) that navigating with a paper map is prone to errors. In contrast to the study by Ishikawa et al. all of our participants were able to reach the destinations.

The participants rated the paper map significantly more helpful for choosing the route to the destination. This goes conform to our findings that the participants spend in average more time interacting with the paper map than with the tactile belt. For maintaining a sense of orientation the ratings for the paper map's and the tactile belt's helpfulness were at a par. There was no significant preference for one of the navigation aids.

The higher appreciation of the belt by females (in terms of usage and perceived helpfulness) indicates that the belt might support the females' way findings strategies better that the males' strategies. According to Lawton [6] females and males tend to use different wayfinding strategies. Men are more likely to use an orientation strategy: monitoring the relative location of reference points in the environment to preserve the sense of orientation. Females, in contrast, are more likely to report a route strategy: getting from one decision point to the other by following turning instructions. Thus, considering it helpful to be aware of a destination's direction and employing a route strategy, which is more likely for females, might be correlated.

\section{CONCLUSIONS}

All roads lead do Rome! However, the question remains how efficient we find our way. Paper maps are a common means for pedestrians to navigate towards a target destination, though people find it difficult to interpret them. In this paper, we presented and approach that adds a tactile cue to the information of the paper map. With our vibrotactile belt the direction of the target destination is displayed in addition to the map. The expectation hypothesis was that the additional tactile display simplifies the interpretation process and positively affects the navigation with a paper map.

For validating our individual hypotheses, we conducted a field study where participants had to navigate through a village with a paper map with and without the tactile display. The participants navigated more effectively by taking shorter routes, they needed to study the map less often, and they lost their orientation less often. These results indicate that the participants were more confident about their route choices. They explored small side paths more often, and were less likely to loose that confidence in case of unexpected problems.

The results allow the conclusion that the tactile information of the direction gives the pedestrians more confidence when navigating towards a target destination. Especially, when it becomes difficult at unclear intersection, the tactile display gives the pedestrian decision support. Providing hands-free continuous and concurrent direction information the pedestrians just have more time to enjoy the route.

Given the technology and infrastructure to communicate a wealth of geographic data in intelligent, context-aware ways we should start thinking of going beyond the paper map 
and route instructions metaphors employed by today's navigation systems.

\section{ACKNOWLEDGMENTS}

The authors are grateful to the European Commission which co-funds the IP HaptiMap (FP7-ICT-224675). We like to thank our colleagues for sharing their ideas with us.

\section{REFERENCES}

[1] A. Aretz and C. Wickens. The mental rotation of map displays. Human Performance, 5(4):303-328, 1992.

[2] I. Aslan, M. Schwalm, J. Baus, A. Krüger, and T. Schwartz. Acquisition of spatial knowledge in location aware mobile pedestrian navigation systems. In MobileHCI '06: Proceedings of the 8th conference on Human-computer interaction with mobile devices and services, pages 105-108, New York, NY, USA, 2006. ACM.

[3] M. Denis, P.-E. Michon, and A. Tom. Applied Spatial Cognition: From Research to Cognitive Technology, chapter Assisting Pedestian Wayfinding in Urban Settings: Why References to Landmarks are Crucial in Direction-Giving, pages 25-52. Psychology Press, 2006.

[4] S. Hurtle and M. Sorrows. Applied Spatial Cognition: From Research to Cognitive Technology, chapter Navigation in Electronic Environments, pages 103-126. Psychology Press, 2006.

[5] T. Ishikawa, H. Fujiwara, O. Imai, and A. Okabe. Wayfinding with a gps-based mobile navigation system: A comparison with maps and direct experience. Journal of Environmental Psychology, 28(1):74-82, March 2008.

[6] C. A. Lawton. Gender differences in way-finding strategies: Relationship to spatial ability and spatial anxiety. Sex Roles, 30(11-12):765-779, Jun 1994.

[7] G. Leshed, T. Velden, O. Rieger, B. Kot, and P. Sengers. In-car gps navigation: engagement with and disengagement from the environment. In $\mathrm{CHI}$ '08: Proceeding of the twenty-sixth annual SIGCHI conference on Human factors in computing systems, pages 1675-1684, New York, NY, USA, 2008. ACM.

[8] R. W. Lindeman, J. L. Sibert, E. Mendez-Mendez, S. Patil, and D. Phifer. Effectiveness of directional vibrotactile cuing on a building-clearing task. In $\mathrm{CHI}$ '05: Proceedings of the SIGCHI conference on Human factors in computing systems, pages 271-280, New York, NY, USA, 2005. ACM.

[9] A. J. May, T. Ross, S. H. Bayer, and M. J. Tarkiainen. Pedestrian navigation aids: information requirements and design implications. Personal and Ubiquitous Computing, 7(6):331-338, 2003.

[10] S. K. Nagel, C. Carl, T. Kringe, R. Märtin, and P. König. Beyond sensory substitutionŮlearning the sixth sense. Journal of Neural Engineering, 2(4):R13+, December 2005.

[11] M. Pielot, N. Henze, W. Heuten, and S. Boll. Evaluation of continuous direction encoding with tactile belts. In HAID '08: Proceedings of the 3rd international workshop on Haptic and Audio Interaction Design, pages 1-10, Berlin, Heidelberg, 2008. Springer-Verlag.
[12] D. A. Ross and B. B. Blasch. Wearable interfaces for orientation and wayfinding. In Assets '00: Proceedings of the fourth international ACM conference on Assistive technologies, pages 193-200, New York, NY, USA, 2000. ACM.

[13] T. Ross, A. May, and S. Thompson. The use of landmarks in pedestrian navigation instructions and the effects of context. In MobileHCI '04: Proceedings of the 6th conference on Human-computer interaction with mobile devices and services, pages 300-304, 2004.

[14] E. Rukzio, M. Müller, and R. Hardy. Design, implementation and evaluation of a novel public display for pedestrian navigation: the rotating compass. In CHI '09: Proceedings of the 27th international conference on Human factors in computing systems, pages 113-122, New York, NY, USA, 2009. ACM.

[15] W. Seager and D. S. Fraser. Comparing physical, automatic and manual map rotation for pedestrian navigation. In CHI '07: Proceedings of the SIGCHI conference on Human factors in computing systems, pages 767-776, New York, NY, USA, 2007. ACM.

[16] N. J. J. M. Smets, G. M. te Brake, M. A. Neerincx, and J. Lindenberg. Effects of mobile map orientation and tactile feedback on navigation speed and situation awareness. In MobileHCI '08: Proceedings of the 10th international conference on Human computer interaction with mobile devices and services, pages 73-80, New York, NY, USA, 2008. ACM.

[17] H. Z. Tan and A. Pentland. Tactual displays for wearable computing. In $I S W C$ '97: Proceedings of the 1st IEEE International Symposium on Wearable Computers, page 84, Washington, DC, USA, 1997. IEEE Computer Society.

[18] Tsukada and Yasumura. Activebelt: Belt-type wearable tactile display for directional navigation. In UbiComp '04: Sixth International Conference on Ubiquitous Computing, 2004.

[19] J. B. F. Van Erp. Guidelines for the use of vibro-tactile displays in human computer interaction. In Proceedings of Eurohaptics 2002, 2002.

[20] J. B. F. Van Erp. Presenting directions with a vibrotactile torso display. Ergonomics, 48:302-313, 2005.

[21] J. B. F. Van Erp, E. L. Groen, and J. E. Bos. A tactile cockpit instrument supports the control of self-motion during spatial disorientation. Human Factors: The Journal of the Human Factors and Ergonomics Society, 48:219-228, 2006.

[22] J. B. F. Van Erp, H. A. H. C. van Veen, C. Jansen, and T. Dobbins. Waypoint navigation with a vibrotactile waist belt. ACM Transactions on Applied Perception, 2(2):106-117, 2005.

[23] H. A. H. C. Van Veen, M. Spapé, and J. B. F. Van Erp. Waypoint navigation on land: Different ways of coding distance to the next waypoint. In Proceedings of the 4 th International Conference EuroHaptics 2004, 2004.

[24] C. Wickens. Multiple resources and performance prediction. Theoretical Issues in Ergonomics Science, 3(2):159-177, 2002. 